
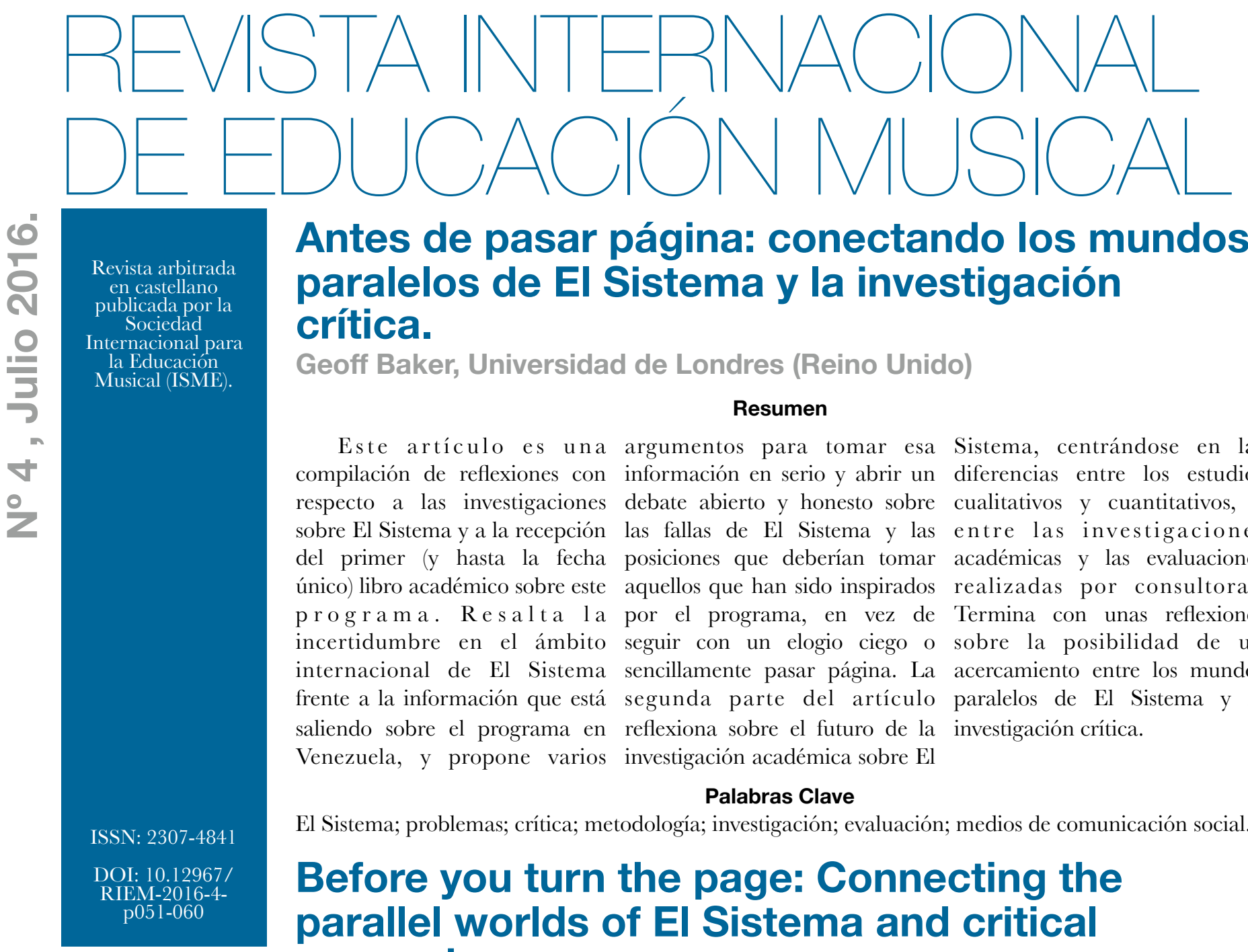

\title{
Antes de pasar página: conectando los mundos paralelos de El Sistema y la investigación crítica.
}

\author{
Geoff Baker, Universidad de Londres (Reino Unido)
}

Resumen

Este artículo es una argumentos para tomar esa Sistema, centrándose en las compilación de reflexiones con información en serio y abrir un diferencias entre los estudios respecto a las investigaciones debate abierto y honesto sobre cualitativos y cuantitativos, y sobre El Sistema y a la recepción las fallas de El Sistema y las entre las investigaciones del primer (y hasta la fecha posiciones que deberían tomar académicas y las evaluaciones único) libro académico sobre este aquellos que han sido inspirados realizadas por consultoras. programa. Resalta la por el programa, en vez de Termina con unas reflexiones incertidumbre en el ámbito seguir con un elogio ciego o sobre la posibilidad de un internacional de El Sistema sencillamente pasar página. La acercamiento entre los mundos frente a la información que está segunda parte del artículo paralelos de El Sistema y la saliendo sobre el programa en reflexiona sobre el futuro de la investigación crítica.

Venezuela, y propone varios investigación académica sobre El

\section{Palabras Clave}

El Sistema; problemas; crítica; metodología; investigación; evaluación; medios de comunicación social.

\section{Before you turn the page: Connecting the parallel worlds of El Sistema and critical research.}

\section{Geoff Baker, University of London (United Kingdom)}

This article consists of a taking that information seriously El Sistema, focusing on the series of reflections on research and beginning an open and differences between qualitative on El Sistema and the reception honest debate about El Sistema's and quantitative studies, and of the first (and to date only) problems and the positions that between academic research and academic book on the program. those who have been inspired by consultancy evaluations. It It underlines the uncertainty of the program might take with concludes with some reflections the international Sistema sphere regard to them, rather than on the possibilities for closing the in the face of the information continuing with blind eulogy or gap between the parallel worlds that is emerging about the turning the page. The second of El Sistema and critical Venezuelan program, and part of the article considers the research. proposes various reasons for future of academic research on

\section{Keywords}

El Sistema; problems; criticisms; methodology; research; evaluation; media.

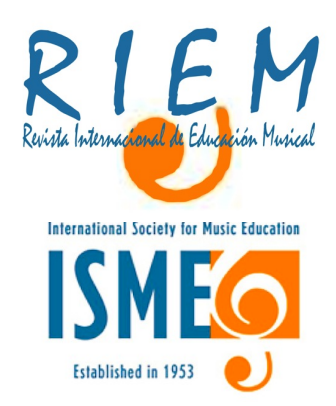




\title{
Antes de pasar página: conectando los mundos paralelos de El Sistema y la investigación crítica.
}

\author{
por Geoffrey Baker, Universidad de Londres (Reino Unido)
}

Al salir del último concierto de la Orquesta Sinfónica Simón Bolívar (OSSB) en el Royal Festival Hall de Londres a principios de 2016, el periodista Damian Thompson se vio sorprendido al ver que un puesto de Foyles estaba vendiendo cedés de Gustavo Dudamel y la OSSB junto a mi libro. En su reseña decía: "subtitulado Orquestando a la fuventud Venezolana, [el libro] examina en detalle todas las acusaciones de abuso sexual, maniobras políticas y engaño musical realizadas en contra del misterioso 'sistema'. Uno se pregunta cuántos miembros de la audiencia, los cuales apenas han terminado de aplaudir, siquiera le echaron una ojeada"l. Esta imagen nos muestra un interesante microcosmos sobre la situación actual: dos mundos paralelos -la industria de El Sistema y la investigación crítica- sentados uno al lado del otro, a la vista de todos, pero con serias tensiones entre ambos que el público en general apenas capta.

No puedo evitar preguntarme en primer lugar cómo es que el libro estaba a la venta allí. El concierto era una situación ventajosa para todos: la industria musical y el público aman El Sistema. ¿Qué espacio podría tener un estudio crítico en este contexto?, ¿cuántos miembros de la audiencia se podrían interesar en leer un libro como éste?, ¿está destinado a ser dejado sobre la mesa, a la vista y recomendado en la contraportada por expertos internacionalmente reconocidos (Lucy Green y Robert Fink), pero al mismo tiempo ignorado por el público en general?

Lo que sigue es una compilación de mis reflexiones sobre esta clase de preguntas y sobre la recepción del primer (y hasta la fecha único) libro académico sobre El Sistema, basado en varios años de un minucioso estudio del programa venezolano.

\section{¿Qué hacer con respecto a Venezuela?}

Cuando El Sistema: Orquestando a la fuventud Venezolana fue publicado a finales de 2014, hubo una reacción inmediata por parte de periodistas y defensores del programa que cuestionaron su veracidad, sus argumentos e incluso mi motivación y honestidad. Pese a que el libro fue evaluado por pares y basado en un exhaustivo estudio realizado en Venezuela, y las críticas vinieron principalmente de personas que tenían muy poco conocimiento sobre el país o su educación musical, las respuestas negativas obtuvieron mucha visibilidad en los medios. Dieciocho meses después, el panorama ha cambiado. Las acusaciones de que el estudio tenía bases teóricas o metodológicas poco sólidas han sido desacreditadas gracias a una serie de reseñas académicas positivas y al respaldo de otros profesionales de ese ámbito, mientras que la veracidad del contenido ha sido corroborada tanto de forma privada como públicamente por músicos venezolanos, siendo el más importante de éstos el violinista Luigi Mazzocchi (Scripp, 2015). Estudios posteriores realizados por otros académicos no solo han validado las críticas escritas en el libro, sino que también las han profundizado y extendido ${ }^{2}$. Es por eso que ahora el debate debe trasladarse a otro plano, y es allí donde surge una trascendental pregunta: ¿qué hacer con esta información sobre El Sistema?, ¿continuar negándola, ignorarla, o enfrentarse a ella?

En el ámbito internacional de El Sistema parece estar surgiendo un proceso de diferenciación. En el extremo más progresista, Venezuela ha comenzado a esfumarse de la vista. La voz más respetada y persuasiva en este círculo es la de Jonathan Govias, quien ha asegurado que "el futuro de El Sistema se encuentra fuera de Venezuela"3. La impresión de que el debate se ha trasladado hacia el norte, de que la gente inspirada por El Sistema ya no siente la necesidad de hablar sobre Venezuela, fue evidente en el reporte de la reunión de Sistema Fellows de enero de 2016 en Boston, en el cual no se hace ninguna mención sobre Venezuela, Abreu o Dudamel, y en la ausencia de temas y disertantes venezolanos en el congreso Reframing El Sistema en Baltimore en abril de $2016^{4}$. Se percibe un deseo de pasar página con respecto a Venezuela. No obstante, en el otro extremo del espectro, el idolatrar al programa venezolano y sus líderes continúa siendo algo común y evidente. En el foro Sistema Global, por ejemplo, muchas personas parecen seguir negando las recientes revelaciones sobre Venezuela, o simplemente no se han informado sobre ellas o han rehusado hacerlo; el resultado de esto es que la fantasía utópica de El Sistema aún sigue teniendo peso. Allí encontramos que los pronunciamientos públicos de Abreu y Dudamel son tratados y repetidos como palabras de gurúes y el programa venezolano es considerado como el modelo a seguir en el mundo.

Aunque tengo mucho más aprecio por las posiciones del ala progresista, me preocupa que en ninguno de los dos extremos exista un debate abierto y honesto sobre las fallas de El Sistema, o sobre las posiciones sobre las mismas que toman aquellos que han sido inspirados por el programa. Yo diría que la urgencia de seguir avanzando también debe ser balanceada con una mirada al pasado, de lo contrario el optimismo y constructividad puede caer en negación y encubrimiento, y las oportunidades para aprender de los errores se verán disminuidas. Actualmente, por lo menos en el debate público, las fallas casi no son abordadas (siendo el blog de Jonathan Govias prácticamente la única excepción), ni existe una discusión detallada sobre los estudios que las examinan -y este enfoque plantea cuestiones tanto éticas como pragmáticas-.

Por ejemplo, aún no he visto ninguna discusión pública sobre la evidente desigualdad de género que existe en la élite de El Sistema, como la distribución 80:20 entre hombres y mujeres en la Orquesta Simón Bolívar o la ausencia de directoras (tanto musicales como administrativas). De igual manera, el tema de las relaciones sexuales entre profesores (por lo general hombres) y estudiantes (por lo general mujeres) apenas ha sido tocado. Luigi Mazzochi confirmó a Scripp (2015) que esta práctica era algo normal dentro de El Sistema, como yo lo había descubierto durante mi investigación de campo. Ha habido un silencio total por parte 
del ámbito de El Sistema sobre este serio problema. La esfera inspirada por este programa tampoco ha podido lidiar con la contradicción entre su discurso generalmente progresista y el hecho de que su padre fundador, José Antonio Abreu, es un acérrimo conservador y un ultra disciplinario que en el pasado ha estado aliado con políticos de derecha de dudosa reputación. Se afirma que su proyecto forma ciudadanos, pero no cuenta con ninguna de las estructuras o procesos democráticos que son centrales para la ciudadanía (Baker, en prensa) y depende de la explotación (bajos salarios y beneficios y derechos laborales mínimos) de gran parte de su fuerza laboral. ¿Realmente estos no son temas de interés ni de importancia para los miembros progresistas en el ámbito inspirado por El Sistema?

De todas maneras, es razonable cuestionar si efectivamente la esfera inspirada por El Sistema debería abordar estos temas. ¿Por qué no pasar página? Después de todo, estos son problemas venezolanos: ¿es necesario que los extranjeros se involucren en esta discusión?

En cierta manera, la respuesta es no. Existe muy poca presión externa sobre la esfera de El Sistema para tratar temas como estos. La prensa, la industria de la música, los bancos de desarrollo y los gobiernos están enamorados de El Sistema. No está en los intereses de las instituciones e individuos que ya se han declarado hinchas del programa, y que incluso han puesto grandes cantidades de dinero en el mismo, el promover o difundir un debate crítico que pueda socavar sus propias acciones y convicciones. Considerando que el pensamiento crítico es muy poco atractivo para los actores más poderosos, la investigación académica puede ser ignorada sin problemas, y esto es principalmente lo que está sucediendo hoy en día a ese nivel institucional.

Sin embargo, Venezuela no solo está lejos, "por allá”», también está «por aquí», en forma de visitas de las más prestigiosas orquestas de El Sistema, en especial la Simón Bolívar. Los testimonios de miembros actuales y pasados de esta orquesta indican que existen importantes interrogantes sobre cómo es manejada ${ }^{5}$. ¿Se deberían ignorar estos problemas y recibir a la orquesta como a la realeza a donde quiera que vaya?, ¿son realmente modelos ideales para los niños de Europa y América del Norte las personas que han llegado a la cima de este sistema altamente competitivo y autoritario, gobernado por la palanca [o tráfico de influencias], el jalado de bolas [o servilismo] y una intolerancia total a la crítica? La predisposición de la esfera de El Sistema de ignorar las verdades incómodas sobre la orquesta y el programa detrás de ella envían un mensaje inequívoco, incluso si son solo pocos los que lo escuchan: olvídense de la «acción social por la música», esto se trata simplemente de la música.

Sin embargo, también existen razones éticas por las cuales no podemos simplemente olvidarnos de Venezuela, sin importar si está «por aquí» o no. La búsqueda de la justicia social exige una clara identificación de la injusticia social, donde sea que esté teniendo lugar, por el bien de aquellos que la sufren y de aquellos que, aunque no la estén sufriendo, estén en riesgo de ser víctimas de ella. Cada día de silencio que pasa es un día desperdiciado en lo que respecta a mejorar las protecciones de los niños dentro de El Sistema. El programa no muestra señales de identificar o tratar la clase de problemas de los cuales se dice está plagado, de modo que el ámbito internacional de El Sistema podría jugar un rol importante en este caso, alentando, e incluso presionando, a la organización para que finalmente lo haga. Además, los problemas que se manifiestan en El Sistema no son para nada exclusivos del mismo, y el traer a la luz los asuntos de discriminación, opresión y hostigamiento en la educación musical podría ser provechoso para futuros estudiantes de música en lugares lejos de Venezuela, al igual que dentro de dicho país. Una investigación decidida sobre los abusos sexuales dentro de El Sistema podría, por ejemplo, contribuir a un naciente movimiento internacional para hacer más segura la educación musical especializada e intensiva para niñas y mujeres jóvenes ${ }^{6}$, y participar en los esfuerzos de hace ya tiempo por comprender y mejorar la problemática dinámica de poder que ha sido encontrada históricamente en muchas instituciones de educación musical especializada ${ }^{7}$.

Es por eso que, en este caso, la esfera de El Sistema tiene una responsabilidad ética, y es una responsabilidad de la cual no se está haciendo cargo al negarse a reconocer o denunciar injusticias. Actualmente, esta esfera está promoviendo la marca de El Sistema y, a través de su silencio público, está apoyando de manera implícita todo lo que esto conlleva. En este sentido, el ámbito internacional de El Sistema es cómplice de las injusticias cometidas en Venezuela, y pasar página sin hablar públicamente sobre esos problemas simplemente perpetúa su culpa por asociación.

Esta esfera dice que está «inspirado» en El Sistema y ha adoptado con gusto el nombre de este programa y la filosofía, publicidad y acceso a financiamiento que ello conlleva. Es por eso que no puede ignorar los problemas dentro de su programa madre. No puede ignorar el hecho de que los participantes de El Sistema compararon a Abreu no con Mahatma Ghandi ni con Nelson Mandela, como los periódicos quieren hacer creer, sino con Adolf Hitler; en un mensaje reciente, un profesor de uno de los núcleos más conocidos de Venezuela comparó el programa con la Juventud Hitleriana. Los amigos músicos de Luigi Mazzocchi le advirtieron que jamás volvería a trabajar en Venezuela si permitiera a Larry Scripp utilizar su nombre real. Mazzocchi habla sobre la «excomunión» de músicos que no obedecieron a Abreu y en un debate en el congreso RIME (Research In Music Education) de 2015, un investigador habló sobre los «refugiados de El Sistema» en el exterior. Estas son revelaciones muy extraordinarias en el contexto de un programa de educación musical. Necesitamos llamar a las cosas por su nombre: la «acción social por la música» que El Sistema promueve es una forma de fascismo social y cultural. Este tipo de verdad incómoda es demasiado grande como para ser ignorada por la esfera internacional de El Sistema, la cual no puede declarar una dedicación a la justicia social al mismo tiempo que opera bajo un estandarte que representa opresión social.

También existen muy buenas razones pragmáticas para que la esfera de El Sistema no pase página tan rápidamente con Venezuela. Y es que el ámbito internacional de El Sistema es como una ciudad construida sobre una falla. En un principio, la gente no se dio cuenta de que existía porque no era visible en la superficie, así que comenzaron a construir sobre ella. Pero pocos años después vino un geólogo y dijo: "están asentados sobre una falla". La mayoría de los residentes respondió diciendo: "yo no veo ninguna falla, te la estás imaginando", y continuaron construyendo. Más adelante, otros geólogos vinieron y dijeron: "no, de verdad 
están construyendo sobre una falla". Este es el punto en el que se encuentra la esfera de El Sistema hoy en día. En pocas palabras, tiene dos opciones: puede continuar ignorando las advertencias y seguir adelante como lo ha estado haciendo, o puede hacer caso a las exhortaciones y reconsiderar qué y cómo está construyendo para poder crear algo más robusto que pueda soportar un temblor de alta magnitud en el futuro.

No hay duda de que nadie sabe exactamente cuándo vendrá este temblor o qué tan fuerte será, e incluso habrá algunos que digan que este temblor nunca vendrá. Pero si un urbanista adoptara este enfoque, estaría tomando un riesgo demasiado grande, y en caso de que un temblor de importante magnitud tuviera lugar, su reputación profesional se vería manchada y hasta podría ser considerado criminalmente responsable por el hecho.

Por lo tanto, la pregunta clave que quiero plantear no es si debiéramos, ni cómo, modificar el modelo conservador venezolano para convertirlo en uno europeo o norteamericano más progresista; la interrogante es qué es lo que van a hacer sobre el hecho de que están sobre una falla. ¿Van a deshacerse de los geólogos?, ¿o van a hacer los edificios de la ciudad resistentes a los terremotos?

\section{Aprender de los errores de El Sistema}

Como sugiere la metáfora del urbanista, sería un error ver a la investigación crítica como algo negativo, pues puede llevar a resultados positivos si es utilizada de manera adecuada. Se puede aprender mucho al observar cuidadosamente los errores cometidos en Venezuela. Utilizando una analogía diferente, es a través del examen minucioso de los accidentes de avión que el transporte aéreo se ha vuelto tan extraordinariamente seguro -no ha sido gracias a incesantes halagos a los pilotos-. La investigación crítica puede entonces ser vista no solo como algo beneficioso, sino que como algo esencial.

El pensar críticamente sobre El Sistema nos permite explorar las debilidades al igual que las fortalezas de las estructuras institucionales autoritarias, caudillistas e impuestas desde arriba. Examinar sus prácticas y valores abre importantes debates sobre pedagogía, currículos, disciplina, inclusión social y democracia. Al enfrentarnos a la variedad de problemas que surgen en el contexto de El Sistema, nos vemos obligados a pensar en cómo evitarlos y buscar soluciones. Nos lleva a preguntarnos cuáles son las mejores formas de promover la solidaridad, la creatividad y la justicia social a través de la educación musical.

A menudo, los principios de El Sistema se basan en creencias problemáticas y no comprobadas. Por ejemplo, muchas de las afirmaciones sociales que son hechas con relación a la orquesta sinfónica no están respaldadas por ningún estudio y, de hecho, muchos de ellos las contradicen. Constantemente se repite la noción de que la orquesta es un motor para el trabajo en equipo, cuando en realidad es considerada por académicos (y muchos músicos profesionales) como un ejemplo de un equipo disfuncional (p. ej. Faulkner, 1973; Hackman, 2002) ${ }^{8}$. La idea de que la orquesta es un modelo para la sociedad entra en conflicto con las ideas modernas sobre sistemas complejos adaptables, de los cuales un conjunto de jazz, por ejemplo, es más representativo (Barrett, 1998).

Por eso los estudios críticos son importantes para todos los que trabajan en el ámbito de El Sistema, impulsando a los profesionales a pensar más a fondo sobre el trabajo en equipo y sobre la educación musical. Si no se toma en serio al pensamiento crítico, se corre el riesgo de hacer más daño que bien. El pensamiento crítico abre las puertas a la idea de que la orquesta sinfónica no es "un modelo para una sociedad global ideal”, como asegura Dudamel (Lee, 2012), sino que más bien puede ser "el modelo de expresión cultural más anti social"9. Es sólo desde aquí, y no desde la complacencia acrítica de Dudamel, que los esfuerzos para transformar a la orquesta en "un vehículo de gran potencial para el cambio social" 10 pueden partir.

También es esencial lidiar con las características problemáticas de El Sistema para así evitar reproducirlas internacionalmente. Por ejemplo, es sorprendente que el ex Sistema Fellow que añadió leves críticas a El Sistema como una adenda al reporte de Scripp (2015) lo hiciera de manera anónima. Al negar que el miedo a hablar públicamente sea tan prevalente en el programa venezolano, los defensores de El Sistema lo han reproducido en Norteamérica. Una aversión al debate crítico y una disposición a reprimir en lugar de encarar ideas desafiantes se ha convertido en algo generalizado en el mundo de El Sistema, y el surgimiento de la censura y la auto-censura dentro de la esfera de El Sistema en América del Norte debería servir como una clara señal de advertencia de que esta esfera está cayendo en la misma trampa que su madre venezolana y se deberían tomar medidas urgentes al respecto ${ }^{11}$.

Existen muchas otras dinámicas constructivas que pueden surgir de un escrutinio crítico de El Sistema. Por ejemplo, sin duda existen lecciones importantes que aprender de los numerosos observadores serios, considerados y experimentados que no comprendieron bien El Sistema y no pudieron ver la serie de injusticias sociales que lleva consigo. Esto nos dice que la justicia social en la educación musical es difícil incluso de identificar, más aún de producir, y que es un tema complejo. Incluso algo tan simple como el proveer a los niños pobres de lecciones de música puede ser un acto de justicia social desde una perspectiva, pero un acto de injusticia desde otra, si se basa en la suposición de que el niño no tiene cultura y es pobre como resultado de esto, o que la justicia económica es un objetivo inalcanzable y la música juega simplemente un rol compensatorio (véase Bates, 2016; Baker, 2016). La investigación crítica nos obliga a examinar cómo observamos, qué es lo que ignoramos fácilmente y cómo podemos ver de manera más aguda; cómo formamos nuestras opiniones sobre la educación musical y cómo nuestras creencias previas pueden ser reforzadas por la propaganda institucional y exhibiciones espectaculares. Parece ser demasiado fácil el asumir que niños haciendo música + declaraciones de buenas intenciones $=$ acción social por la música.

Las investigaciones sugieren que la complacencia es un peligro en casos como éste: una creencia exagerada que el poder del hacer música de cualquier tipo tenga algún efecto transformacional en los jóvenes y el ignorar que la educación musical tiene el potencial de acarrear costos e incluso de causar daño (Bowman, 2009; Gould, 2009; Bradley, 2015; Matthews, 2015). Todos estos son temas importantes de discusión para los educadores musicales, y mientras más sean compartidos y debatidos, mayores serán las chances de evitar este tipo de errores en programas inspirados en El Sistema y también será más fácil alcanzar resultados positivos. Lo que 
sería tremendamente negativo es que una esfera impulsada por deseos de inclusión o justicia ignore los debates contemporáneos sobre los mismos, repitiendo de esta manera - los errores del pasado.

\section{¿Cuál es el futuro de la investigación académica sobre El Sistema?}

Pareciera haber dos tendencias generales en la investigación sobre El Sistema en la actualidad. Una de ellas es principalmente cualitativa y se enfoca en preguntas generales -sociales, políticas, económicas, culturales- aunque también trabaja a través de la observación estrecha de prácticas y discursos. La otra es más cuantitativa y se enfoca más en los resultados académicos y psicosociales (p. ej. Osborne y otros, 2015). Ambos tienen un rol importante que cumplir, y los estudios más ambiciosos incluyen ambos enfoques, pero hay dos inquietudes que me gustaría resaltar aquí. Una de ellas es que el movimiento defensor de El Sistema y sus aliados institucionales tienden a ignorar el primer enfoque y sólo discutir el segundo. La otra es que estas dos corrientes corren el riesgo de seguir como dos monólogos en vez de un diálogo.

Es importante observar que las principales afirmaciones sobre El Sistema venezolano son muy generales y ambiciosas: son hechas en el plano de lo ético, espiritual y macro-social, y tienen que ver con valores y carácter. También se enfocan de manera específica en el poder de la música para derrotar a la pobreza. La misión de El Sistema es oficialmente el "rescate pedagógico, ocupacional y ético de la infancia y la juventud, mediante la instrucción y la práctica colectiva de la música, dedicada a la capacitación, prevención y recuperación de los grupos más vulnerables del país" ${ }^{12}$. Según su visión, el programa se centra en el "desarrollo integral del ser humano" y cultiva "valores transcendentales que inciden en la transformación del niño, el joven y el entorno familiar", ofreciendo "una oportunidad para el desarrollo personal en lo intelectual, en lo espiritual, en lo social y en lo profesional, rescatando al niño y al joven de una juventud vacía, desorientada y desviada"l3. Por esta razón los estudios académicos también tienen que tocar estas áreas (aunque no solamente éstas) y comprobar estas afirmaciones, que forman la base de la fama global del proyecto, de hecho es aquí en donde se han enfocado los principales estudios cualitativos y en donde se encuentran las principales críticas. No hay dudas de que es valioso e interesante saber si El Sistema ha traído consigo efectos positivos en otras áreas, pero éste no es el terreno marcado por El Sistema y discutido por sus críticos. Los debates más fuertes sobre el programa no se centran en si aprender música mejora la habilidad cognitiva de los individuos o su desempeño académico, sino más bien en temas de política, moral y ética -clase, neoliberalismo, neocolonialismo- y si la educación debiera ser vista en términos de salvación, ofreciendo, según asegura Abreu, una riqueza espiritual que sobrepasa a la pobreza material.

Es así que una evaluación detallada de El Sistema no puede ser reducida a la medición de los logros de los estudiantes o incluso su entusiasmo, sino que también debe tomar en consideración temas políticos y estructurales más amplios. Por tomar un ejemplo diferente pero relacionado, el debate sobre la educación privada no gira en torno a si las escuelas privadas benefician o gustan a quienes asisten a ellas o no. O tomen el caso de La Tercera Ola, un experimento realizado en una escuela Secundaria en California en 1967 por un profesor de historia llamado Ron Jones ${ }^{14}$. El experimento produjo mejorías inmediatas en disciplina, motivación y logros académicos. ¿Por qué no fue, entonces, aplicado a más escuelas? Porque se trataba de un experimento con el fascismo. El estudiar La Tercera Ola solamente a través de métodos cuantitativos llevaría a proponer un sistema de educación fascista. Por eso los resultados educativos no pueden tomarse en cuenta si los aspectos sociales, políticos y éticos se dejan aparte.

Por ejemplo, ¿se puede justificar la creación y mantenimiento de costosos programas en contextos en los cuales el financiamiento de otras formas de educación musical y para otros servicios sociales se esté reduciendo, a veces de manera dramática?, ¿están los programas de El Sistema financiados por gobiernos como los del Reino Unido actuando como una cortina de humo o un «velo de cultura» (Logan, 2016) para distraer la atención de la sostenida reducción de provisiones sociales y educativas por parte del Estado bajo la excusa de la austeridad?, ¿está El Sistema, con sus afirmaciones sin comprobar y francamente improbables sobre sus milagrosos efectos sobre la pobreza (Fink, 2016) redirigiendo recursos de programas más efectivos contra la pobreza y enmascarando en lugar de resolviendo los problemas sociales estructurales con proyectos paliativos altamente selectivos pero que se venden bien con la prensa?

En ese caso, una pregunta importante no es si la educación musical es buena para los niños (una afirmación generalmente aceptada), sino más bien si la educación musical de El Sistema -la cual está basada en métodos e ideas de instrucción de música clásica claramente anticuados- es el mejor modelo para los niños y la sociedad en general y cuáles son los costos asociados que trae a los niveles micro y macro sociales. En el nivel micro, es esencial considerar cómo las características específicas de El Sistema -a diferencia de otras formas de aprendizaje musical- pueden tener un efecto en los resultados. Por ejemplo, los efectos cognitivos de aprender música son generalmente aceptados; aun así, como Levine y Levine (1996) demuestran, la falta de control puede llevar a que los músicos orquestales experimenten altos niveles de estrés, lo que puede llevar a una reducción en las habilidades cognitivas. De esta manera, el ambiente orquestal puede anular algunos de los beneficios del aprendizaje musical, convirtiéndola en una herramienta ineficiente en la búsqueda de objetivos cognitivos.

Un artículo reciente sobre música y empatía (Rabinowitch, 2012) demuestra cómo el aprendizaje musical puede mejorar la empatía, pero no habla de cualquier tipo de aprendizaje musical, sino más bien, de un programa especialmente diseñado para la interacción musical en grupo, el cual incluye juegos y tareas ${ }^{15}$. En otras palabras, es probable que los tipos de experiencias que surgen del aprendizaje musical estén relacionadas con el tipo de valores que se les inyecta. El Sistema no habla de empatía, habla de disciplina, obediencia y buen comportamiento. Es así que aquí podemos ver uno de los riesgos que existen al trasladar El Sistema de Venezuela a otros contextos: es un modelo diseñado, sobre todo, para inculcar valores sociales específicos relacionados al conformismo, y si otros valores sociales son más importantes en el contexto nuevo -tales como la empatía, la creatividad o la autonomía- entonces puede que sea el modelo equivocado. Vale la pena resaltar 
que este experimento sobre la empatía "enfatizó repetidamente que no había ningún objetivo que alcanzar a través de las interacciones aparte de jugar juntos y disfrutar de la interacción" (ibíd., p. 83). El Sistema, por el contrario, tiene un enfoque extremadamente orientado a los resultados, de hecho, es una de los elementos que más lo diferencian. En Venezuela, todo gira en torno al concierto. Si la empatía o la creatividad es un resultado deseable importante, ¿por qué escoger El Sistema y no otra forma de educación musical? ${ }^{16}$

Otro paso importante a dar por los investigadores en este área es una interacción crítica más profunda no solo con programas basados en El Sistema sino también con otros estudios de los mismos. Un tema que requiere especial atención son las evaluaciones realizadas por consultoras, las cuales son un género distinto al de la investigación académica. Según Logan $\left(2015^{\text {b }}\right)$, las evaluaciones oficiales de los programas de El Sistema plantean tantas preguntas como las que contestan ${ }^{17}$. Una tarea importante que queda por delante es la de analizar más a fondo la brecha que existe entre los estudios académicos y las evaluaciones de consultoría, brecha que es evidente, por ejemplo, cuando se comparan los estudios realizados por Allan y otros (2010), Borchert (2012), Rimmer, Street y Phillips (2014), Logan (2015a; 2016), y Bull (2016), los cuales presentan una imagen ambivalente de los programas inspirados por El Sistema en el Reino Unido y los lisonjeros reportes oficiales como Evaluation of Big Noise (Scottish Government Social Research, 2011). ¿Cómo es posible que todos los estudios académicos tienen importantes reservas sobre el tema, mientras ninguna de las evaluaciones oficiales produce algo similar?

Más recientemente, en diciembre de 2015, se publicó una evaluación de El Sistema Aotearoa que fue preparada para el Ministerio de Cultura y Patrimonio de Nueva Zelanda y la Orquesta Filarmónica de Auckland por parte de una consultora evaluadora de estudios (McKegg y otros, 2015). Este reporte fue muy bien recibido por la gente que estaba involucrada en la esfera de El Sistema a nivel internacional, pero uno no puede evitar preguntarse si hubo alguien que analizó con detalle la sección de metodología.

El reporte indica que "se utilizó un enfoque de adaptación de casos exitosos (Brinkerhoff, 2003) con la utilización de una investigación apreciativa (Preskill \& Catsambas 2006)" (McKegg y otros, 2015, p. 9). En otras palabras, solo los estudiantes más sobresalientes fueron incluidos en el estudio, aquellos que fueron identificados por los líderes y profesores del programa como los más activos o comprometidos, o quienes mostraban una mejora más sustancial ya sea en lo social o en lo musical, centrándose la investigación en sus experiencias más positivas.

Este es un método de investigación algo marginal. No se menciona en el libro más importante sobre metodologías en esta área, el Case Study Research de Robert K. Yin (2009). Es abordado brevemente por Stufflebeam y Coryn (2014), quienes indican que tiene ciertas fortalezas, como identificar lo que funciona bien, transmitir tranquilidad a los patrocinadores y levantar la moral, además de llevar poco tiempo y ser barato. No obstante, tiene debilidades obvias: es muy limitado y no así exhaustivo, y tiene un enfoque cortoplacista. "Esta limitación es considerada como una debilidad solo si un estudio que utiliza el Método de Casos de Éxito es interpretado erróneamente como una evaluación exhaustiva del mérito y valor de un programa" (ibíd., p.
142-3), algo que era prácticamente inevitable una vez que el reporte llegara a la esfera de El Sistema y los matices metodológicos fueran pulidos con declaraciones generalizadas sobre el éxito del programa.

Existen otros problemas más comunes que el reporte mismo menciona como advertencia, como la falta de datos de referencia, lo que quiere decir que "es posible que la diferencia que hemos observado existe debido a que los estudiantes con muchos logros sean más propensos a seguir participando del programa" (McKegg y otros, 2015, p. 17), una conclusión que de hecho se lleva bien con el estudio de Rimmer, Street y Phillips (2014) sobre El Sistema en Inglaterra. El estudio se basa en «el juicio general del profesor», lo que supone reconocer que es en parte subjetivo, y no toma en cuenta los estudiantes que abandonaron el programa (una laguna común en estudios de El Sistema). Es decir, hay razones suficientes para cierto nivel de escepticismo. Pero el tema de fondo es que el reporte, mientras que puede ser una herramienta útil para quienes tengan intereses en el programa, no sirve como una evaluación exhaustiva del mismo.

La predisposición de los defensores de El Sistema de celebrar un reporte que se enfoca solo en los aspectos positivos plantea un interrogante interesante en lo que respecta a la recepción de mi libro, ya que varias figuras de ese campo desestimaron mi estudio, incluso llegando a decir que ni siquiera se trataba de investigación, ya que no mostraba una imagen «equilibrada» y que una investigación rigurosa debía serlo. No obstante, los autores del reporte sobre El Sistema Aotearoa indicaron lo siguiente:

Está bien documentado que es posible aprender mucho sobre un programa al enfocarse ya sea en casos de éxito o fracaso -es decir, los casos más extremos-. Estos casos más extremos arrojan resultados singulares y ofrecen lecciones que pueden ser aplicadas en aspectos más típicos de un programa o servicio (McKegg y otros, 2015, p. 9).

Lo que es bueno para el ganso, es bueno para la gansa. Si un reporte basado en casos exitosos es permisible, entonces también lo es uno enfocado en fracasos. La única «regla» es que la metodología debe ser claramente especificada y no se debe insinuar que los resultados son exhaustivos, tal y como lo resalté en la página 20 de mi libro (Baker, 2014):

Este libro no es una narrativa completa ni concluyente, sino un análisis crítico e informado sobre las afirmaciones y actores principales de El Sistema, resaltando las preguntas actuales y los debates sobre Abreu y su programa.

\section{¿Podrían acercarse entre sí los mundos paralelos?}

La justificación para seguir investigando es clara, pero es igualmente importante abordar mucho más los estudios que ya existen. Actualmente ya hay suficientes estudios publicados como para iniciar debates importantes, pero éstos por lo general son débiles o ausentes, al menos en la esfera pública. Esta situación presenta difíciles interrogantes sobre el espacio que existe para la investigación crítica en la esfera de El Sistema y el valor de llevarla a cabo.

El Sistema tiene lazos con importantes intereses comerciales e institucionales. Es un pilar esencial de las marcas de Dudamel, la OSSB, la Filarmónica de Los Ángeles, el Centro Southbank, y otros. Es por esto que cualquier debate sobre las ventajas y desventajas del programa educacional siempre correrá el riesgo de ser 
eclipsado por cuestiones promocionales y comerciales. Tales instituciones e intereses especiales que están comprometidos con el modelo de El Sistema parecen estar más interesados en argumentos que justifiquen una postura que ya han tomado que en la investigación en sí misma. Además, los medios de comunicación puede desviar o ahogar la conversación en cualquier momento. El inusual nivel de atención que le han dado los medios (para tratarse de un tema de educación musical) ha atraído a figuras mediáticas al debate, pero que por lo general tienen una comprensión limitada del tema. Malentendidos fundamentales, ya sean accidentales o deliberados, han sido consagrados como verdades y muchos descubrimientos relevantes simplemente han sido ignorados. Periodistas que han elogiado a El Sistema en el pasado no están generalmente abiertos a descubrimientos que puedan socavar sus opiniones públicas.

Esto no presenta un panorama alentador para un académico crítico. El trabajar como investigador en medio de este tipo de fuerzas es como ser una pulga sobre la piel de un elefante. De hecho, habría que preguntarse si tan siquiera es posible tener un debate serio, profundo e informado en un contexto como este, en el que existe una narrativa fuertemente arraigada desde hace ya un buen tiempo que es aceptada por instituciones y en la cual muchas personas creen apasionadamente. La investigación crítica siempre es vulnerable ante las «historias milagrosas», historias que son más digeribles y memorables que la investigación y que los programas, marcas y audiencias prefieren escuchar. ¿Existe espacio para que estudios disidentes sean reconocidos y tengan un impacto en este contexto, fuera del mundo académico, o es esta una esperanza inútil?

El problema no es solo el reducido espacio para la labor académica crítica, sino que también lo es la gran brecha que existe entre los estudios académicos y la industria de El Sistema y sus patrocinadores. Este abismo es ilustrado por las reacciones que provocó mi libro. Las reseñas en Amazon pueden ser vistas como indicación de esto: todas son o de cinco estrellas (el máximo) o de una (el mínimo), no hay punto medio. Esta dramática bifurcación ha sido reproducida en reseñas tanto impresas como en redes sociales, en las cuales periodistas de alto perfil y defensores de El Sistema han llegado a conclusiones diametralmente opuestas a las de académicos internacionalmente reconocidos. Mi trabajo ha sido descrito como «peligrosamente deficiente» por el periodista Mark Swed y también como «el libro de música de nuestros tiempos» por el experto en educación musical Randall Everett Allsup (2016). Nicholas Kenyon, el director del Centro Barbacana, lo consideró «muy poco convincente»; Luigi Mazzochi, una ex estrella de El Sistema, dijo que "daba justo en el clavo" (Scripp, 2015, p. 8) (dicho sea de paso, es fascinante leer que las experiencias de los músicos venezolanos parecen "muy poco convincentes" escrito por una persona que sabe muy poco de Venezuela. A veces la ficción es más fácil de creer que la verdad). Existen muchos otros ejemplos como este, y son una indicación de que nos enfrentamos a mucho más que simples diferencias de opinión; aquí existe un abismo entre visiones muy apasionadas sobre la música, la educación y la sociedad, entre dos mundos paralelos y distantes.

¿Hay alguna esperanza de cerrar esta brecha?, ¿pueden la investigación y las publicaciones escritas tener algún efecto sobre esta situación? Más aun, ¿quién leerá este artículo que estoy escribiendo en este momento?, ¿existe alguna posibilidad de que siquiera llame la atención de alguien, por no hablar de ser tomado en cuenta, en esa distante orilla?

Por decirlo de otra manera, ¿qué tendría que suceder para que Swed, Kenyon y el lobby de los defensores de El Sistema cambien una opinión que han hecho pública de manera tan contundente? Después de todo, existe evidencia de que "cuando las personas están mal informadas, el entregarles los hechos para que corrijan esos errores solo tiene el efecto de hacer que se aferren más tenazmente a sus creencias" ${ }^{18}$. El debate sobre el cambio climático es un claro ejemplo de esto, aunque también lo es el apoyo inquebrantable del que disfruta Donald Trump. Como Thomas L. Friedman observó recientemente, la mayoría de los votantes en los Estados Unidos toman sus decisiones según sus corazonadas, no en detalles o en hechos ${ }^{19}$. La esfera de El Sistema parece tener aspectos en común y por esta razón no veo claramente el sentido de continuar trabajando en El Sistema. No ha habido ningún cuestionamiento significativo de mi libro en el mundo académico, así que la necesidad de acumular más evidencia o continuar argumentando mi posición dentro de esta esfera es mínima; y es poco probable que la resistencia proveniente de afuera del mundo académico vaya a cambiar sin importar los hechos, detalles o argumentos que yo presente.

No obstante, aún tengo la esperanza de que los mundos paralelos se acerquen. Cuando he tenido la oportunidad de interactuar con personas en los niveles medios y bajos del campo inspirado en El Sistema -profesores de música, administradores, Sistema Fellows- me he encontrado con gente agradable y de mente abierta que está dispuesta a conversar y debatir, y sentí que hubo un genuino intercambio de opiniones y experiencias. No estoy hablando de una muestra significativa, y el hecho de que la mayoría de este tipo de reuniones haya tenido lugar en congresos académicos sugiere que puede que no sea muy representativa; pero me ha llevado a creer que, detrás de las batallas de alto perfil en los periódicos y revistas, sí puede existir un diálogo productivo entre el movimiento de El Sistema y el mundo académico crítico, pese a que actualmente se vea limitado por unas cuantas figuras de autoridad miopes. Asistir al congreso Reframing El Sistema me afianzó en esta creencia, como cuando tuve conversaciones estimulantes con varios profesores, investigadores y directores del ámbito inspirado por El Sistema. Parece que el apetito para el debate está creciendo poco a poco.

También me da esperanza el hecho de que el director de un proyecto inspirado en El Sistema -uno de los críticos más duros de mi libro cuando éste fue publicado- ha entablado un diálogo conmigo sobre mi investigación con una mente abierta, y hemos llegado a construir una relación amigable y productiva. No pensamos igual, pero las diferencias se han vuelto constructivas en lugar de destructivas. Este cambio, debo resaltar, no surgió porque yo haya tenido algún cambio de opinión o después de que le haya dorado la píldora. Llegó a ser así porque mi interlocutor se sentó a leer y pensar sobre mi libro como debe ser. Esta experiencia me lleva a concluir que los mundos de El Sistema y la investigación crítica no solo podrían cruzarse, sino que deberían hacerlo. Esto sería mucho más productivo y éticamente sensato para la esfera internacional de El Sistema que seguir con su elogio ciego de Venezuela o el querer pasar página. 


\section{Notas}

${ }^{1}$ http://www.spectator.co.uk/2016/01/was-barenboimhappy-hiding-inside-a-provincial-orchestra-from-venezuela/. ${ }^{2}$ Véase la edición especial de Action, Criticism \& Theory for Music Education, 15(1) (enero de 2016); también Logan 2015a. ${ }^{3}$ http://jonathangovias.com/2015/11/08/what-next-forsistema/.

${ }^{4}$ http://sistemafellows.typepad.com/my-blog/2016/02/ looking-to-the-future.html.

${ }^{5}$ Véase http:/ / es.tocarypensar.com/blog/dentro-de-laorquesta-sinfnica-simn-bolvar. Ver además Baker (2014) y Scripp (2015).

${ }^{6}$ Véase por ejemplo https://ianpace.wordpress.com y también http://www.historyandpolicy.org/news/article/ music-education-and-abuse-of-children-and-young-people, sobre el seminario celebrado el 15 de septiembre de 2015 en el Institute of Musical Research de Londres.

${ }^{7}$ La bibliografía sobre este tema es extensa: véase, p. ej., Baker (2014).

${ }^{8}$ Véase también http://www.theguardian.com/lifeandstyle/ 2016/apr/09/what-im-really-thinking-orchestral-musician.

${ }^{9} \mathrm{http}: / /$ jonathangovias.com/2015/04/05/this-is-where-weflew/.

${ }^{10}$ Ibíd.

${ }^{11}$ Véase http://es.tocarypensar.com/blog/censura-yautocensura-en-la-esfera-de-el-sistema al respecto.

${ }^{12}$ http://fundamusical.org.ve/category/el-sistema/mision-yvision/.

13 Ibíd.

${ }^{14}$ http://libcom.org/history/the-third-wave-1967-accountron-jones.

${ }^{15}$ Este artículo fue resaltado por Jonathan Govias.

${ }^{16}$ Véase por ejemplo Laurence (2008).

${ }^{17}$ Véase también Baker (2014), capítulo 11.

${ }^{18}$ http://www.alternet.org/media/most-depressing-

discovery-about-brain-ever. Para una visión más académica, véase https://www.researchgate.net/publication/

295478583_Making_The_Truth_Stick_and_The_Myths_Fa de_Lessons_from_Cognitive_Psychology.

${ }^{19}$ http://www.nytimes.com/2016/03/09/opinion/only-

trump-can-trump-trump.html

\section{Referencias citadas}

Allan, J., Moran, N., Duffy, C., y Loening, G. (2010). Knowledge exchange with Sistema Scotland. Fournal of Education Policy, 25(3), 335-347.

Allsup, R. E. (2016). The enchanted empire: an essay review of El Sistema Orchestrating Venezuela's Youth by Geoffrey Baker. Music Education Research, 1-3. doi: 10.1080/14613808.2016.1138663

Baker, G. (2014). El Sistema: Orchestrating Venezuela's youth. Nueva York: Oxford University Press.

Baker, G. (2016). Editorial introduction: El Sistema in critical perspective. Action, Criticism, and Theory for Music Education 15(1), 10-32. Disponible en http://act.maydaygroup.org/ articles/Baker15_1.pdf.

Baker, G. (en prensa). Citizens or subjects? El Sistema in critical perspective. En D. Elliott, M. Silverman y W. Bowman (Ed.), Oxford Handbook of Artistic Citizenship. Nueva York: Oxford University Press.
Barrett, F. J. (1998). Creativity and improvisation in jazz and organizations: Implications for organizational learning. Organization Science, 9(5), 605-622.

Bates, V. C. (2016). Foreword: How can music educators address poverty and inequality? Action, Criticism, and Theory for Music Education 15(1), 1-9. Disponible en http:// act.maydaygroup.org/articles/Bates 15_1.pdf.

Borchert, G. (2012). Sistema Scotland: A critical inquiry into the implementation of the El Sistema model in Raploch. Trabajo Fin de Máster, Universidad de Glasgow. Disponible en http://theses.gla.ac.uk/ 4044/1/2012BorchertMMus.pdf.

Bowman, W. D. (2009). No one true way: Music education without redemptive truth. En T. Regelski y T. Gates (Ed.), Music Education for Changing Times: Guiding Visions for Practice. Dordrecht: Springer.

Bradley, D. (2015). Hidden in plain sight: Race and racism in music education. En C. Benedict, P. Schmidt, G. Spruce, y P. Woodford (Ed.), Oxford Handbook of Social Justice in Music Education. Nueva York: Oxford University Press.

Bull, A. (2016). El Sistema as a bourgeois social project: Class, gender, and Victorian values. Action, Criticism, and Theory for Music Education 15(1), 120-53. Disponible en http://act.maydaygroup.org/articles/Bull15_1.pdf.

Faulkner, R. (1973). Career concerns and mobility motivations of orchestra musicians. Sociological Quarterly, 14 (3), 334-349.

Fink, R. (2016). Resurrection Symphony: El Sistema as ideology in Venezuela and Los Angeles. Action, Criticism, and Theory for Music Education 15(1), 33-57. Disponible en http://act.maydaygroup.org/articles/Fink15_1.pdf.

Gould, E. (2009). Disorientations of desire: Music education queer. En T. Regelski y T. Gates (Ed.), Music Education for Changing Times: Guiding Visions for Practice. Dordrecht: Springer.

Hackman, R. (2002). Leading teams: Setting the stage for great performances. Boston: Harvard Business School Press.

Kenyon, N. (2015). The triumph of a musical adventure. The New York Review of books. Reseña de El Sistema: Orchestrating Venezuela's Youth, por Geoffrey Baker. Disponible en http: / /www.nybooks.com/articles/2015/09/24/ venezuela-triumph-musical-adventure/

Laurence, F. (2008). Music and empathy. En O. Urbain (Ed.), Music and conflict transformation: Harmonies and dissonances in geopolitics. Londres: I. B Tauris.

Lee, C. (2012). Bravo, Gustavo! How Maestro Dudamel is saving classical music. Newsweek, 6 de febrero. Disponible en http://www.newsweek.com/bravo-gustavo-howmaestro-dudamel-saving-classical-music-65809.

Levine, S. y Levine, R.. (1996). Why they're not smiling: Stress and discontent in the orchestra workplace. Harmony, 2, 15-25.

Logan, O. $\left(2015^{\mathrm{a}}\right)$. Doing well in the eyes of capital: Cultural transformation from Venezuela to Scotland. En J. A. McNeish, A. Borchgrevink y O. Logan (Ed.) Contested powers: The politics of energy and development in Latin America. Londres: Zed Books.

Logan, O. $\left(2015^{\mathrm{b}}\right)$. Hand in glove: El Sistema and neoliberal research. Disponible en https://www.researchgate.net/ publication/287202150. 
Logan, O. (2016). Lifting the veil: A realist critique of Sistema's upwardly mobile path. Action, Criticism, and Theory for Music Education 15(1), 58-88. Disponible en http:/ /act.maydaygroup.org/articles/Logan15_1.pdf.

Matthews, R. (2015). Beyond toleration-facing the other. En C. Benedict, P. Schmidt, G. Spruce, y P. Woodford (Ed.), Oxford Handbook of Social Justice in Music Education. Nueva York: Oxford University Press.

McKegg, K., Crocket, A., Goodwin, D. y Sauni, P. (2015). Sistema Aotearoa outcome evaluation. Kinnect Group. Disponible en http://www.mch.govt.nz/sites/default/ files / Sistema $\% 20$ Aotearoa $\% 20$ outcome $\% 20$ evaluation \%20Dec\%202015\%20\%28D-0644229\%29_0.PDF.

Osborne, M. S., McPherson, G. E., Faulkner, R., Davidson, J. W. y Barrett, M. S. (2015). Exploring the academic and psychosocial impact of $\mathrm{El}$ Sistema-inspired music programs within two low socio-economic schools. Music Education Research, 18(2), 156-175.

Rabinowitch, T. C. (2012). Musical games and empathy. Education and Health, 30(3), 80-84.

Rimmer, M., Street, J. y Phillips, T. (2014). Understanding the cultural value of In Harmony-Sistema England. Arts \& Humanities Research Council/University of East Anglia. Disponible en https://ueaeprints.uea.ac.uk/50664/4/ Rimmer_et_al_2014_Understanding_the_Cultural_Valu e_of_In_Harmony_Sistema_England_final_pdf.

Scottish Government Social Research (2011). Evaluation of Big Noise, Sistema Scotland. Edimburgo: Crown. Disponible en h t t p : / / w w w.gov.s c ot / Res o u r e / D o c / 345409/0114922.pdf.

Scripp, L. (2015). The need to testify: A Venezuelan musician's critique of El Sistema and his call for reform. Disponible en http://www.researchgate.net/publication/285598399.

Stufflebeam, D. L. y Coryn, C. L. S. (2014). Evaluation theory, models, and applications. San Francisco: Jossey-Bass.

Yin, R. K. (2009). Case study research: Design and methods. Londres: Sage. 


\section{Sobre el Autor}

\section{Geoff Baker}

Geoff Baker es Catedrático en el Departamento de Música en el Royal Holloway de la Universidad de Londres y Director del Instituto de Investigacion Musical. Entre sus libros se incluyen Imposing Harmony: Music and Society in Colonial Cuzco (Duke, 2008), que ganó el premio Robert Stevenson de la Sociedad Musicológica de los Estados Unidos; Buena Vista in the Club: Rap, Reggaetón, and Revolution in Havana (Duke, 2011); y El Sistema: Orchestrating Venezuela's Youth (OUP, 2014). Fue co-investigador del proyecto Growing Into Music del programa Beyond Text del Consejo de Investigación en Artes y Humanidades (AHRG) del Reino Unido, por medio del que hizo una serie de películas sobre el aprendizaje musical infantil en Cuba y Venezuela, así como investigador asociado del proyecto del Consejo Europeo de Investigación (ERG) titulado Music, Digitisation, Mediation: Towards Interdisciplinary Music Studies.

Más información sobre el autor y su obra en http:// es.tocarypensar.com

\section{Geoff Baker}

Music Department, Royal Holloway University of London Egham, Surrey TW20 0EX (Reino Unido) geoff.baker@rhul.ac.uk

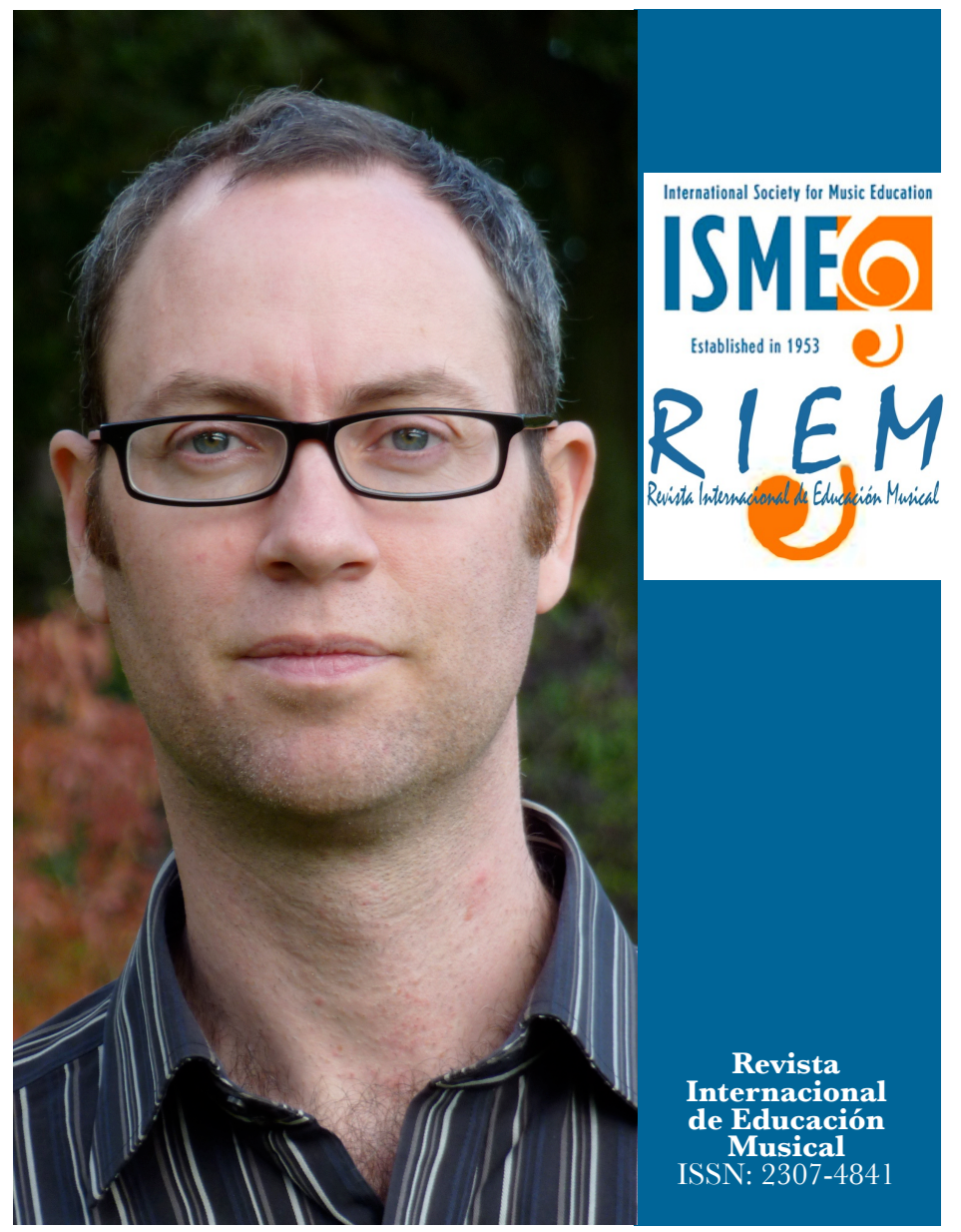

\section{EQUPO EDITORIAL}

Editor:

José Luis Aróstegui Plaza, Universidad de Granada (España)

\section{Editora Adjunta:}

Rosa María Serrano Pastor, Universidad de Zaragoza (España)

\section{Consejo Editorial}

Carlos Abril, Universidad de Miami (Estados Unidos)

María del Carmen Aguilar, Instituto Coral de Buenos Aires (Argentina)

Miquel Alsina Tarrés, Universidad de Gerona (España)

Carmen Carrillo Aguilera. Universidad Internacional de Cataluña (España)

Sergio Luiz Figueiredo, Universidad del Estado de Santa Catarina (Brasil)

Patricia Adelaida González, Universidad Autónoma de Chihuahua (México)

Claudia Gluschankof, Instituto Levinsky (Israel)

Josep Gustems Carnicer, Universidad de Barcelona (España)

María Cecilia Jorquera Jaramillo, Universidad de Sevilla (España)

Gotzon Ibarretxe Txakartegi, Universidad del País Vasco (España)

Ana Laucirica Larrinaga, Universidad Pública de Navarra (España)

Ana Lucia Louro, Universidad Federal de Santa María (Brasil)
Isabel Cecilia Martínez, Universidad Nacional de La Plata (Argentina)

Teresa Mateiro, Universidad del Estado de Santa Catarina (Brasil)

María Teresa Moreno, Universidad Laval (Canadá)

Graça Boal Palheiros, Instituto de Educación de Oporto (Portugal)

Jèssica Pérez Moreno, Universidad Autónoma de Barcelona (España) y Universidad de Londres (Reino Unido)

Gabriel Enrique Rusinek Milner, Universidad Complutense de Madrid (España)

Patrick K. Schmidt, Universidad de Ontario Occidental (Canadá)

Favio Shifres, Universidad Nacional de La Plata (Argentina)

Maria dels Àngels Subirats Bayego, Universidad de Barcelona (España)

António Ângelo Ferreira Vasconcelos, Instituto de Educación de Setúbal (Portugal)

Maria Helena Vieira, Universidad del Miño (Portugal)

Gloria Patricia Zapata Restrepo, Universidad de Antioquía (Colombia) 